\title{
Magnetic Impurities in Mott-Hubbard Antiferromagnets
}

\author{
Avinash Singh ${ }^{1,2}$ and Prasenjit Sen $^{2}$ \\ ${ }^{1}$ Theoretische Physik III, Universität Augsburg, 86135 Augsburg, Germany \\ ${ }^{2}$ Department of Physics, Indian Institute of Technology, Kanpur 208016, India
}

\begin{abstract}
A formalism is developed to treat magnetic impurities in a Mott-Hubbard antiferromagnetic insulator within a representation involving multiple orbitals per site. Impurity scattering of magnons is found to be strong when the number of orbitals $\mathcal{N}^{\prime}$ on impurity sites is different from the number $\mathcal{N}$ on host sites, leading to strong magnon damping and singular correction to low-energy magnon modes in two dimensions. The impurity-scattering-induced softening of magnon modes leads to enhancement in thermal excitation of magnons, and hence to a lowering of the Néel temperature in layered or three dimensional systems. Weak impurity scattering of magnons is obtained in the case $\mathcal{N}^{\prime}=\mathcal{N}$, where the impurity is represented in terms of modified hopping strength, and a momentum-independent multiplicative renormalization of magnon energies is obtained, with the relative magnon damping decreasing to $q^{2}$ for long-wavelength modes. Split-off magnon modes are obtained when the impurity-host coupling is stronger, and implications are discussed for twomagnon Raman scattering. The mapping between antiferromagnets and superconductors is utilized to contrast formation of impurity-induced states.
\end{abstract}

71.27.+a, 75.10.Jm, 75.10.Lp, 75.30.Ds

\section{INTRODUCTION}

While the problem of static impurities in antiferromagnetic insulators is more than twenty five years old, [1] it has attracted renewed attention after the discovery of high- $\mathrm{T}_{\mathrm{c}}$ cuprate superconductors, [2] since their parent compounds are antiferromagnetic insulators. From the very early days of high- $T_{c}$ superconductivity a number of doping studies have been done with various static impurities - both magnetic, [3] and nonmagnetic [4 6 ] replacing copper from the $\mathrm{Cu}-\mathrm{O}$ planes as in $\mathrm{La}_{2} \mathrm{CuO}_{4}$. Susceptibility measurements in high- $\mathrm{T}_{\mathrm{c}}$ cuprates doped with magnetic impurities like $\mathrm{Fe}, \mathrm{Ni}$, Co give evidence of local-moment formation, 傮 which is expected to be intrinsically associated with the magnetic impurities. This is unlike the case of nonmagnetic impurities such as Zn, $\mathrm{Al}$, Ga etc. which, despite being intrinsically nonmagnetic, give rise to local moments in the copper-oxide planes when doped in cuprate antiferromagnets. This was inferred earlier from the Curie-Weiss behavior of the magnetic susceptibility, [4, 7] and has been recently confirmed in the Y-NMR studies of doped 1-2-3 systems as seen in the progressively increasing linewidth of the YNMR signal with decreasing temperature. [6,8] Xiao et $a l$. have also ascertained the spin states of different magnetic dopants from the observed local moments, [3] and find, for example, that $\mathrm{Fe}$ is in a spin- $\frac{5}{2}$ state, whereas $\mathrm{Ni}$ is in a spin- 1 state. They also find a correlation between $\mathrm{T}_{\mathrm{c}}$ reduction and size of the local moment, consistent with the magnetic pair breaking mechanism.

Although theoretically the problem of magnetic impurities in an antiferromagnet has been studied recently within the Heisenberg representation of localized spins, [9] no such comprehensive study exists within the MottHubbard model, which provides a good description of the
$3 \mathrm{~d}$ holes in the $\mathrm{Cu}-\mathrm{O}$ planes of high- $\mathrm{T}_{\mathrm{c}}$ superconductors. Recently the problem of nonmagnetic impurities in the Mott-Hubbard antiferromagnet was addressed and defect states, local-moment formation, impurity-scattering of magnons, and finite-temperature magnetic dynamics in layered systems were studied. [10,11] Other recent works on static vacancies in antiferromagnets include exact diagonalization studies with Heisenberg model, [12] linear spin wave theory, [13] and exact diagonalization of strongly correlated small clusters. 14 While nonmagnetic impurities can be simply represented by spinindependent impurity potential, the situation is more complex for magnetic impurities. In this paper we introduce several representations to treat magnetic impurities in different situations. A simple extension to spindependent impurity potential is followed by a more sophisticated approach involving a generalized $\mathcal{N}$-orbital Hubbard model with multiple orbitals per site. Broadly there are two distinct classes depending on whether the number of orbitals $\mathcal{N}^{\prime}$ at the impurity site is the same as or different from the number of orbitals $\mathcal{N}$ at the host sites. In the case $\mathcal{N}^{\prime}=\mathcal{N}$ the magnetic impurity is represented through a modified hopping strength $t^{\prime}$ between the impurity orbitals and the neighboring host orbitals. In the strong-correlation limit $(U \gg t)$ wherein the MottHubbard AF with $\mathcal{N}$ orbitals per site maps to the spin $S=\mathcal{N} / 2$ quantum Heisenberg AF, the modified hopping strength translates into modified exchange coupling $J^{\prime}=4 t^{2} / U$ between the impurity spin and the neighboring host spins. This describes the situation where, in spin language, the impurity spin $S^{\prime}$ is equal to the host spin $S$, but is coupled to its neighbors with a different exchange interaction $J^{\prime}$. Similarly the case $\mathcal{N}^{\prime} \neq \mathcal{N}$ with no modification in hopping strength or Hubbard interaction energy corresponds to the situation where the impurity 
spin is different from the host spins $\left(S^{\prime} \neq S\right)$.

\section{SINGLE-ORBITAL MAGNETIC IMPURITY}

In this section we consider a single-orbital magnetic impurity embedded in an AF host which is described by the Hubbard model with one orbital per site with exactly half filling. For concreteness we consider the square lattice, generalization to other bipartite lattices being straightforward. The host Hamiltonian is

$$
H_{0}=-t \sum_{\langle i j\rangle \sigma}\left(a_{i \sigma}^{\dagger} a_{j \sigma}+a_{j \sigma}^{\dagger} a_{i \sigma}\right)+U \sum_{i} n_{i \uparrow} n_{i \downarrow},
$$

where $t$ is the nearest-neighbor (NN) hopping strength and $U$ the on-site Coulomb repulsion. The AF state and its associated features such as sublattice magnetization, magnon energies, quantum corrections etc. have been studied earlier in detail. 15. We model the single-orbital impurity in terms of locally modified hopping term $t^{\prime}$ between the impurity orbital and its NN host orbitals. The Hamiltonian with such an impurity on site $I$ can be written as below, where the sum is over all nearest neighbors $J$ of the impurity sites $I$, and $\delta t=t^{\prime}-t$ is the hopping perturbation around the impurity site,

$$
H=H_{0}+\delta t \sum_{\langle I J\rangle \sigma}\left(a_{I \sigma}^{\dagger} a_{J \sigma}+a_{J \sigma}^{\dagger} a_{I \sigma}\right)
$$

We start with the perturbative method where the impurity-induced perturbation $\left[\delta \chi^{0}\right] \equiv\left[\chi^{0}\right]-\left[\chi_{\text {host }}^{0}\right]$ to the zeroth-order, antiparallel-spin, particle-hole propagator is obtained in powers of $\delta t / t$, and resulting corrections to its eigenvalues then yield the renormalization in magnon energies. 16 Diagrammatic contributions to $\left[\delta \chi^{0}\right]$ to first order in $\delta t$, and their evaluation in the strong-correlation limit have been discussed earlier in context of the hopping disorder problem. 116] We obtain for the diagonal, off-diagonal, and nearest-neighbor diagonal terms, expressed in units of $-t^{2} / \Delta^{3}$ for convenience,

$$
\left[\delta \chi^{0}\right]_{I I}=\frac{z}{2} \frac{\delta t}{t} ; \quad\left[\delta \chi^{0}\right]_{I J}=\left[\delta \chi^{0}\right]_{J I}=\left[\delta \chi^{0}\right]_{J J}=\frac{1}{2} \frac{\delta t}{t}
$$

where $z=4$ is the coordination number for the square lattice, $2 \Delta \approx U$ is the Hubbard gap, and only terms upto order $\left(t^{2} / \Delta^{3}\right)$ have been retained, appropriate to the strong-correlation limit. We notice that the sum of all matrix elements diagonal in sublattice basis, $\left[\delta \chi^{0}\right]_{I I}+$ $\left[\delta \chi^{0}\right]_{J J}$ is precisely equal to the sum of off-diagonal matrix elements $\left[\delta \chi^{0}\right]_{I J}+\left[\delta \chi^{0}\right]_{J I}$. An immediate consequence of this correlation is that the Goldstone mode is preserved and that generally the effective scattering of low-energy, long-wavelength magnon modes is weak.

If the impurity is on an A-sublattice site, then for the first-order correction we obtain after summing over nearest neighbor terms,

$$
\begin{aligned}
& \delta \lambda_{q}^{(1)} \equiv\left\langle q\left|\left[\delta \chi^{0}\right]\right| q\right\rangle= \\
& \alpha^{2}\left[\delta \chi^{0}\right]_{I I}+\alpha \beta z \gamma_{q}\left[\delta \chi^{0}\right]_{I J}+\beta \alpha z \gamma_{q}\left[\delta \chi^{0}\right]_{J I}+\beta^{2} z\left[\delta \chi^{0}\right]_{J J}
\end{aligned}
$$

where $\alpha$ and $\beta$ are the magnon amplitudes on $\mathrm{A}$ and B sublattices respectively, and $\gamma_{q}=\left(\cos q_{x}+\cos q_{y}\right) / 2$. An identical result is obtained when the impurity is on a B-sublattice site, because in this case $\alpha$ and $\beta$ are simply exchanged in the above equation, and since $\left[\delta \chi^{0}\right]_{I I}=z\left[\delta \chi^{0}\right]_{J J}$, this expression is symmetric under exchange of $\alpha$ and $\beta$. Using $\alpha=\sqrt{\frac{1}{N}\left(1-\omega_{q}^{0}\right)}$ and $\beta=-\sqrt{\frac{1}{N}\left(1+\omega_{q}^{0}\right)}$, where $\omega_{q}^{0}=\sqrt{1-\gamma_{q}^{2}}$ is the host magnon energy in units of $2 J$ for the momentum- $q$ mode, we obtain after summing over contributions from all impurities

$$
\delta \lambda_{q}^{(1)}=x z \frac{\delta t}{t}\left(1-\gamma_{q}^{2}\right)
$$

where $x$ is the total impurity concentration, and impurities are assumed to be evenly distributed between the two sublattices. The renormalized magnon energy, given by the pole in the magnon propagator, is now obtained from the solution of the equation $1-\sqrt{\omega^{2}+\gamma_{q}^{2}}+\delta \lambda_{q}^{(1)}=0$, and upto first order in the effective impurity strength $x \delta t / t$ we obtain

$$
\omega_{q}=\omega_{q}^{0}\left(1+x z \frac{\delta t}{t}\right) .
$$

This result agrees exactly with the calculations [9] on the Heisenberg model in that there are no singular corrections to the magnon energy in the case $S^{\prime}=S$, and the correction is proportional to $x \delta t / t=(1 / 2) x \delta J / J$.

Turning now to the magnon-energy renormalization of the localized, high-energy modes with energy near $2 J$, which correspond to local spin deviation, we have $\alpha=0$, $\beta=1$, so that $\delta \lambda^{(1)}=\frac{1}{2} z(\delta t / t)$. This implies that the magnon energy gets shifted from $2 J$ to

$$
\omega=2 J\left(1+\frac{z}{2} \frac{\delta t}{t}\right) .
$$

In this case the impurity concentration does not enter the magnon-energy renormalization, rather it has a bearing on the spectral weight of these high-energy modes. Thus for $\delta t$ positive, the magnon spectrum goes up by energy $z J \delta t / t$. This increase is expected from the simple picture of these high-energy modes corresponding to local spin deviations. The energy cost of making a spin deviation on the impurity site is $z J^{\prime} / 2$, where $J^{\prime} / 2$ is the bond strength. With $t^{\prime}=t(1+\delta t / t)$, to first order in $\delta t / t$ we have $\Delta \epsilon=z\left(J^{\prime}-J\right) / 2=z J \delta t / t$.

The exact-eigenstates analysis also shows that precisely one magnon state at the upper end of the spectrum is split off from the magnon energy band. These split-off modes are strongly localized around the impurity sites, and hence correspond to local spin deviations. 
Furthermore, for different values of the impurity hopping $t^{\prime} / t$ it is seen from the magnon spectrum that the energy separation of the split-off state from the upper end of the spectrum increases roughly in proportion to $\delta t$, as obtained in the perturbative analysis. This exacteigenstates approach for obtaining magnon energies and wavefunctions from the fermionic eigensolutions in the self-consistent AF state has been described earlier. [17]

\section{SPIN-DEPENDENT IMPURITY POTENTIAL}

Nonmagnetic impurities in the Mott-Hubbard AF were modelled earlier via a spin-independent impurity potential term, and as a natural extension we therefore consider the following spin-dependent impurity term for magnetic impurities,

$$
H_{\mathrm{imp}}^{\mathrm{mag}}=\sum_{I} \Psi_{I}^{\dagger}\left[-\sigma_{3} V\right] \Psi_{I},
$$

where $\Psi_{I}=\left(\begin{array}{ll}a_{I \uparrow} & a_{I \downarrow}\end{array}\right)$. A spin-independent impurity potential $\epsilon_{0}$ can be included for generality, however, we shall consider the limit $V>>\epsilon_{0}$, so that the potential for spin $\sigma$ fermion is $V_{\sigma} \approx-\sigma V$. We choose $V$ to be positive for impurities on the A-sublattice sites, so that $V_{\uparrow}$ is very low and $V_{\downarrow}$ is very high. The sign of $V$ is reversed for impurities on B-sublattice sites. This choice of potential ensures that the magnetization on the impurity sites follows the host AF ordering. Such a spin-dependent impurity potential can arise from a coupling $-\vec{\sigma} . \vec{S}_{\text {imp }}$ between the itinerant fermion spin $\vec{\sigma}$ and the static magnetic impurity spin $\vec{S}_{\text {imp }}$, resulting from a strong Hubbard interaction. Since experiments on high- $\mathrm{T}_{\mathrm{c}}$ cuprates show the impurity spin to be antiferromagnetically coupled with the host spins, [3] we take the local field direction to be along the local magnetization direction $(\hat{z})$. The low potential (for spin-up) is justified in view of the fact that the ionization energy for both $\mathrm{Fe}^{+3}$ and $\mathrm{Ni}^{+2}$ i.e., the fourth and the third ionization energies respectively for $\mathrm{Fe}$ and $\mathrm{Ni}$ are much higher than the third ionization energy for $\mathrm{Cu}$. Whereas the ionization energies for $\mathrm{Fe}^{+3}$ and $\mathrm{Ni}^{+2}$ are $54.8 \mathrm{eV}$ and $35.17 \mathrm{eV}$ respectively, the ionization energy for $\mathrm{Cu}^{+2}$ is $20.2 \mathrm{eV}$.

We now examine formation of impurity-induced states due to this spin-dependent impurity potential. Within the T-matrix analysis, used earlier for nonmagnetic impurities, [10] energies of impurity-induced states are obtained from solutions of $g_{I I}^{\sigma}(\omega)=1 / V_{\sigma}$. For large $|V| / U$ these impurity states are formed at energies $\sim-\sigma V$ for the two spins, and are essentially site localized and therefore decoupled from the system. Thus, for the magneticimpurity case when the impurity spin is antiferromagnetically coupled to the neighboring host spins, a significant difference from the nonmagnetic-impurity case is that there are no defect states formed in the Hubbard gap. Rather only impurity states are formed, far removed in energy from the Hubbard bands.
Within the above representation of magnetic impurities in terms of spin-dependent impurity potential, the fermion number is unchanged, unlike the case of nonmagnetic impurities where one fermion is removed for every added impurity. Hence the impurity sites do not quite act as spin vacancies. Nonetheless, the presence of a impurity potential term which breaks time-reversal symmetry leads to a partial decoupling of the impurity site from the host. This is most easily seen in the limit $V \rightarrow \infty$ where the local antiparallel-spin, particle-hole excitations are suppressed by the large energy difference $2 \mathrm{~V}$, leading to an absence of the $\omega$ term, and therefore to strong magnon scattering. Quite generally, the particle-hole energy difference for antiparallel spins is modified by the spindependent impurity potential from $2 \Delta$ to $2 \Delta+2 V$, leading to a modification in the $\omega$ term. For spin-independent impurity potential the particle-hole energies are shifted equally, and hence it is the removal of a fermion from the impurity site that is crucial. As a result of this decoupling of magnetic impurity sites, a qualitatively identical impurity-induced perturbation $\left[\delta \chi^{0}(\omega)\right]$ is obtained, leading to similar results for magnon renormalization as for the nonmagnetic-impurity case, where singular corrections were obtained for low-energy magnon modes in two dimensions. [11] The strong impurity-scattering of magnons also introduces significant damping, with the ratio of magnon damping term to its energy being simply proportional to the impurity concentration $x$ for longwavelength modes.

\section{GENERALIZED HUBBARD-MODEL REPRESENTATION}

In order to represent higher-spin magnetic impurities, we now generalize to the situation with $\mathcal{N}$ and $\mathcal{N}^{\prime}$ orbitals on host and impurity sites respectively. An appropriate model for this case is the generalized Hubbard model with multiple orbitals per site. This model has been used earlier to study quantum corrections in the antiferromagnetic state in a spin-rotationally-symmetric formalism, where a systematic perturbative expansion in powers of $1 / \mathcal{N}$ was developed. [15. We introduce a slight extension here in this model which makes it equivalent, in the strong correlation limit, to the spin- $S$ QHAF, where $S=\mathcal{N} / 2$. The modification is to allow the NN hopping term to operate between all orbitals, whereas the hopping term considered earlier was diagonal in the orbital index. 115] We therefore consider the following Hamiltonian for the AF host,

$$
\begin{aligned}
H & =-t \sum_{<i j>\sigma \alpha \beta}\left(a_{i \sigma \alpha}^{\dagger} a_{j \sigma \beta}+\text { h.c. }\right) \\
& +\frac{U}{\mathcal{N}} \sum_{i \alpha \beta}\left(a_{i \uparrow \alpha}^{\dagger} a_{i \uparrow \alpha} a_{i \downarrow \beta}^{\dagger} a_{i \downarrow \beta}+a_{i \uparrow \alpha}^{\dagger} a_{i \uparrow \beta} a_{i \downarrow \beta}^{\dagger} a_{i \downarrow \alpha}\right)
\end{aligned}
$$

where $\alpha$ and $\beta$ are the orbital indices which run from 1 to $\mathcal{N}$, and the two Hubbard interaction terms are re- 
spectively direct and exchange type interactions with respect to orbital indices. In the symmetric case when the two interaction strengths are identical, as considered here, the system possesses spin-rotational symmetry. It has been shown earlier that in the symmetric case the two interaction terms can together be written as $H_{\mathrm{int}}=-(U / \mathcal{N}) \sum_{i}\left(\vec{S}_{i} \cdot \vec{S}_{i}+n_{i}^{2}\right)$, where $\vec{S}_{i}$ and $n_{i}$ are the total spin and charge density operators, respectively. Spin-rotational-symmetry is therefore inherent in this impurity representation as well. Furthermore, in the strong correlation limit, a strong Hund's coupling exists which energetically favors the maximum multiplicity case $(S=\mathcal{N} / 2)$ for the total spin operator $\vec{S}_{i}$.

Magnetic impurities are represented by introducing $\mathcal{N}^{\prime} \neq \mathcal{N}$ orbitals at the impurity sites. We first examine the transverse spin fluctuation propagator in the host AF state, $\chi^{-+}\left(r t, r^{\prime} t^{\prime}\right) \equiv\left\langle\Psi\left|S^{-}(r t) S^{+}\left(r^{\prime} t^{\prime}\right)\right| \Psi\right\rangle$, where $\vec{S}(r t)=\sum_{\alpha} \psi_{\alpha}^{\dagger}(r t) \frac{\vec{\sigma}}{2} \psi_{\alpha}(r t)$ is the total spin operator. Again, at the RPA level the magnon propagator is given by $\chi^{0}(\omega) /\left[\mathbf{1}-(U / \mathcal{N}) \chi^{0}(\omega)\right]$, where $\left[\chi^{0}(\omega)\right]$ now involves orbital summations, with matrix elements given by,

$$
\left[\chi^{0}(\omega)\right]_{i j}=i \int \frac{d \omega^{\prime}}{2 \pi} \sum_{\alpha \beta} G_{i \alpha, j \beta}^{\uparrow}\left(\omega^{\prime}\right) G_{j \beta, i \alpha}^{\downarrow}\left(\omega^{\prime}-\omega\right) .
$$

Since each orbital is now connected via hopping to $\mathcal{N}$ orbitals on the NN sites, the electronic spectral weights are correspondingly modified. For example, in the strong-correlation limit, the on-site majority and minority spin densities in each orbital are now $1-\mathcal{N} t^{2} / \Delta^{2}$ and $\mathcal{N} t^{2} / \Delta^{2}$ respectively. A straightforward extension of the earlier analysis in the strong-correlation limit 15 leads to:

$$
\chi^{0}(q, \omega)=\mathcal{N} \frac{1}{U} \mathbf{1}-\mathcal{N}^{2} \frac{D}{2} \frac{t^{2}}{\Delta^{3}}\left[\begin{array}{cc}
1+\frac{\omega}{D J \mathcal{N}} & \gamma_{q} \\
\gamma_{q} & 1-\frac{\omega}{D J \mathcal{N}}
\end{array}\right]
$$

where $J=4 t^{2} / U$ as usual, and $D$ is dimensionality of the hypercubic lattice. Since different orbitals on the same site are not directly coupled, the intrasite propagator is diagonal in orbital index, and therefore the leading order diagonal terms (the $1 / U$ and the $\omega$ term) are proportional to $\mathcal{N}$. However, the NN hopping operates between all orbitals, and therefore the offdiagonal term and the next-to-leading order piece (arising from hopping) in the diagonal term are both proportional to $\mathcal{N}^{2}$. The magnon energies are now given by: $\omega_{q}=D J \mathcal{N} \sqrt{1-\gamma_{q}^{2}}=2 D J S \sqrt{1-\gamma_{q}^{2}}$ in terms of the $\operatorname{spin} S=\mathcal{N} / 2$.

We now introduce a magnetic impurity in the system with $\mathcal{N}^{\prime} \neq \mathcal{N}$ orbitals at the impurity site $I$. The resulting modification in the electronic spectral weights leads to the following changes in the $\left[\chi^{0}(\omega)\right]_{i j}$ matrix elements for $i, j$ in the vicinity of the impurity site $I$ :

$$
\left[\chi^{0}\right]_{I I}=\mathcal{N}^{\prime} \frac{1}{U}-\mathcal{N} \mathcal{N}^{\prime} \frac{D}{2} \frac{t^{2}}{\Delta^{3}}\left(1+\frac{\omega}{D J \mathcal{N}}\right)
$$

$$
\begin{aligned}
{\left[\chi^{0}\right]_{I J} } & =-\mathcal{N N}^{\prime} \frac{D}{2} \frac{t^{2}}{\Delta^{3}} \frac{1}{z} \\
{\left[\chi^{0}\right]_{J J} } & =\mathcal{N} \frac{1}{U}-\mathcal{N}^{2} \frac{D}{2} \frac{t^{2}}{\Delta^{3}}\left(1-\frac{\omega}{D J \mathcal{N}}\right) \\
& -\mathcal{N}\left(\mathcal{N}^{\prime}-\mathcal{N}\right) \frac{D}{2} \frac{t^{2}}{\Delta^{3}} \frac{1}{z}
\end{aligned}
$$

Since now the local Hubbard interaction strength itself is not uniform but depends on the number of site orbitals, we have to multiply the $\left[\chi^{0}\right]$ matrix with the diagonal interaction matrix $[\mathcal{U}]$ containing elements $U / \mathcal{N}$ for host sites and $U / \mathcal{N}^{\prime}$ for the impurity site. We therefore examine the local matrix elements of the matrix product $\left[\mathcal{U} \chi^{0}\right]_{i j}=\mathcal{U}_{i i} \chi_{i j}^{0}$ for $i, j$ in the vicinity of the impurity site. The impurity-induced perturbation in the matrix elements of the product $\left[\mathcal{U} \chi^{0}\right]$ are obtained as below:

$$
\begin{aligned}
\delta\left[\mathcal{U} \chi^{0}(\omega)\right]_{I I} & =0 \\
\delta\left[\mathcal{U} \chi^{0}(\omega)\right]_{I J} & =0 \\
\delta\left[\mathcal{U} \chi^{0}(\omega)\right]_{J I} & =-U\left(\mathcal{N}^{\prime}-\mathcal{N}\right) \frac{D}{2} \frac{t^{2}}{\Delta^{3}} \frac{1}{z} \\
\delta\left[\mathcal{U} \chi^{0}(\omega)\right]_{J J} & =-U\left(\mathcal{N}^{\prime}-\mathcal{N}\right) \frac{D}{2} \frac{t^{2}}{\Delta^{3}} \frac{1}{z} .
\end{aligned}
$$

We now obtain the magnon-energy renormalization by perturbatively obtaining the impurity-induced correction to the eigenvalues of the $\left[\mathcal{U} \chi^{0}(\omega)\right]$ matrix. As discussed earlier, [11] we treat $\delta\left[\mathcal{U} \chi^{0}(\omega)\right]$ as the perturbation matrix, and determine corrections to eigenvalues of $\left[\chi_{\text {host }}^{0}(\omega)\right]$. Evaluating the first-order correction $\left\langle q\left|\delta\left[\mathcal{U} \chi^{0}(\omega)\right]\right| q\right\rangle$ from the magnon eigenvector $|q\rangle$, and retaining terms to first order only, we obtain:

$$
\delta \lambda_{q}^{(1)}=U\left(\mathcal{N}^{\prime}-\mathcal{N}\right) \frac{D}{2} \frac{t^{2}}{\Delta^{3}} \frac{\omega}{D J} .
$$

As for the nonmagnetic impurity case, we obtain here a correction which is linear in energy, and this signifies strong impurity scattering of magnons for longwavelength, low-energy modes, leading to singular corrections in two dimensions and strong magnon damping from second-order scattering processes. [11] The scattering term is explicitly proportional to the difference $\left(\mathcal{N}^{\prime}-\mathcal{N}\right)$ between the number of orbitals on the impurity site and the host sites, which arises from the different dynamics of the impurity spin and the host spins. This generally implies that impurity scattering of magnons is strong when the impurity spin $S^{\prime}=\mathcal{N}^{\prime} / 2$ is different from the host spin $S=\mathcal{N} / 2$, in agreement with earlier studies within the Heisenberg model, [9] and the one-band model with nonmagnetic impurities where $\mathcal{N}=1$ and $\mathcal{N}^{\prime}=0$. 11

\section{CONCLUSIONS}

In conclusion, we have developed a formalism to treat magnetic impurities in a Mott-Hubbard antiferromagnetic insulator within a representation involving multiple orbitals per site. For the case $\mathcal{N}^{\prime}=\mathcal{N}$, when the 
impurity spin is identical to the host spin, the magnetic impurity is represented by locally modified hopping strength, and we find that the effective scattering of long-wavelength magnon modes is weak, leading to momentum-independent multiplicative renormalization of magnon energies. For positive hopping perturbation $\delta t$ we find localized, split-off magnon modes corresponding to local spin deviations at impurity sites. These split-off modes will be relevant in two-magnon Raman scattering which probes high energy magnetic excitations. In the other case $\mathcal{N}^{\prime} \neq \mathcal{N}$, when the impurity spin is different from the host spin, we obtain strong impurity scattering of magnon modes proportional to the difference $\left(\mathcal{N}^{\prime}-\mathcal{N}\right)$, leading to singular corrections in two dimensions and strong magnon damping. The impurity-scattering-induced softening of magnon modes implies enhancement in thermal excitation of magnons, and hence to a lowering of the Néel temperature in layered or three dimensional systems. We also find that the process of putting additional impurity orbitals leads to enhanced impurity magnetization and localization of electronic states at the impurity, indicating partial decoupling of the impurity site from the host. A unique feature of having multiple impurity orbitals is the presence of exactly site-localized eigenstates in the electron spectrum which are completely antisymmetric between impurity orbitals.

When the magnetic impurity is represented in terms of a spin-dependent impurity potential, we find that the breaking of time-reversal symmetry leads to a decoupling of the impurity site from the host, and strong magnon scattering similar to the case of spin vacancies is obtained. We also find that when the magnetic impurity spin is antiferromagnetically coupled to the neighboring host spins, only impurity states are formed, and there are no defect states formed within the Hubbard gap. The local moment associated with the magnetic impurity therefore intrinsically arises from the spin-density difference at the impurity site. Using the well-known particle-hole transformation, The problem of magnetic impurities in an AF can be mapped to that of nonmagnetic impurities in a superconductor, which is characterized by absence of defect states within the superconducting gap and robustness of superconducting gap. [19] Conversely, a nonmagnetic impurity in a positive- $U$ Hubard AF maps onto a magnetic impurity in a negative- $U$ Hubbard superconductor, and here defect states are formed within the gap in both cases. 20,21

\section{ACKNOWLEDGMENTS}

Helpful conversations with S. N. Basu, S. Tewari, D. Sa, V. Subrahmanyam, and V. A. Singh are gratefully acknowledged. This work was supported in part by a Research Grant (No. SP/S2/M-25/95) from the Department of Science and Technology, India. A.S. also acknowledges support from the Alexander von Humboldt Foundation. 
For a review, see R. A. Cowley and W. J. L. Buyers, Rev. Mod. Phys. 44, 406 (1972).

2 J. G. Bednorz and K. A. Müller, Z. Phys. B 64, 188, (1986).

3 G. Xiao, M. Z. Cieplak, J. Q. Xiao, and C. L. Chien, Phys. Rev. B 42, 8752 (1990).

4 G. Xiao, M. Z. Cieplak, A. Gavrin, F. H. Streitz, A. Bakhshai and C. L. Chien, Phys. Rev. Lett, 60, 1446 (1988).

$5 \quad$ R. E. Walstedt, R. F. Bell, L. F. Schneemeyer, and T. V. Waszcazk, Phys. Rev. B 48, 10646 (1993).

6 A. V. Mahajan, H. Alloul, G. Collin, and J. F. Marucco, Phys. Rev. Lett. 72, 3100 (1994).

7 C. -S. Gee et al., J. Superconductivity, 1, 63, (1988).

8 H. Alloul, P. Mendels, H. Casalta, J. F. Marucco, and J. Arabski, Phys. Rev. Lett. 67, 3140 (1991).

9 C. C. Wan, A. B. Harris and D. Kumar, Phys. Rev. B 48, 1036 (1993).

10 P. Sen, S. Basu and A. Singh, Phys. Rev. B 50, (RC) 10381 (1994).

11 P. Sen and A. Singh, Phys. Rev. B 53, 328 (1996).

12 N. Bulut. D. Hone, D. J. Scalapino, and E. Y. Loh, Phys. Rev. Lett. 62, 2192 (1989).

13 W. Brenig and A. Kampf, Phys. Rev. B 43, 12914 (1991).

14 D. Poilblanc, D. J. Scalapino, and W. Hanke, Phys. Rev. Lett. 72, 884 (1994).

15 A. Singh, Phys. Rev. B 43, 3617 (1991).

16 S. Basu and A. Singh, Phys. Rev. B 55, 12338 (1997).

17 S. Basu and A. Singh, Phys. Rev. B 53, 6406 (1996).

18 S. Basu and A. Singh, Phys. Rev. B 54, 6356 (1996).

19 P. W. Anderson, J. Phys. Chem. Solids 11, 26 (1959).

20 H. Shiba, Prog. Theor. Phys. 40, 435 (1968).

21 K. Maki, Superconductivity, Vol II, edited by R. D. Parks (Dekker, New York, 1969). 